\title{
La competitividad en las microempresas en Manta 2019
}

Competitiveness in micro business in Manta 2019

\author{
Autores \\ Gonzalo Andrés Rodríguez Arrieta. Investigador del Senescyt. Manabí. Ecuador. \\ E-mail:profegonzalorodriguez@gmail.com http://orcid.org/0000-0001-6849-106X \\ Evelyn Dyann Cano Lara. Universidad Laica Eloy Alfaro de Manabí. Manabí. Ecuador. \\ E-mail: Evelyn.cano@uleam.edu.ec http://orcid.org/0000-0001-6754-0587.
}

Andrea Lissete Ruiz Velez. Universidad San Gregorio de Portoviejo. Manabí. Ecuador. E-mail:alruiz@sangregorio.edu.ec http://orcid.org/0000-0002-8938-0163

Fecha de recibido: 2019-10-20

Fecha de aceptado para publicación: 2019-12-23

Fecha de publicación: 2019-12-30

\section{Resumen:}

Las microempresas en el Ecuador representan el $90,78 \%$ del total de empresas y constituyen un conjunto de organizaciones de vital importancia para el país, que se destacan por el aporte en lo económico y en el empleo. Pero al igual que cualquier otra empresa, la competencia en un mundo globalizado es el punto clave para lograr la supervivencia y continuidad en el tiempo. El objetivo de la presente investigación es determinar el grado de competitividad de las microempresas dentro del Cantón Manta - Ecuador según la percepción de sus representantes. Por ello se realizó un estudio descriptivo, de base cuantitativo, a fin de medir la percepción de los empresarios de microempresas a través de la adaptación de un instrumento de medición comprobado. Las características y particularidades que se han mostrado a lo largo de esta investigación se complementan en lo teórico y práctico, en cuanto a la informalidad, baja capacitación, poca planificación, dificultades financieras, entre otros. El comportamiento de los datos demuestra una proporción dos a uno en las respuestas de acuerdo con relación a las de en desacuerdo, midiendo de esta manera una competitividad aceptable por parte de las microempresas en Manta.

Palabras Clave: Desarrollo microempresario, competitividad, microempresas en Manta

\begin{abstract}
:
Microenterprises in Ecuador represent $90.78 \%$ of the total number of companies, which means a set of organizations of vital importance for the country, which highlights the contribution in economic terms and employment. But like any other company, competition in a globalized world is the key point for survival and continuity over time. The objective of this research is to determine the degree of competitiveness of microenterprises within the Manta Canton - Ecuador according to the perception of their representatives. Therefore, a quantitative-based descriptive study was carried out to measure the perception of microenterprise entrepreneurs through the adaptation of a proven measuring instrument. The characteristics and particularities that have been shown throughout this research are complemented in theoretical and practical terms, in terms of informality, low training, poor planning, financial difficulties, among others. The behavior of the data demonstrates a two-to-one ratio in the responses according to those in disagreement, thereby measuring acceptable competitiveness by microenterprises in Manta.
\end{abstract}

Keywords: microenterprise development, competitiveness, microenterprises in Manta 


\section{Introducción}

Competir en los sistemas económicos sociales de la actualidad es el desafío más grande que tienen las empresas y de cuyo éxito depende la supervivencia de ellas, aún más en un mundo globalizado. El manejo de recursos financieros, la habilidad de dirigir personas, la diferenciación del producto, el conocimiento de la competencia, la creación verdadera del valor del producto, la fidelización del cliente, entre otras, agregan más presión a lo que otrora era solo saber producir para vender. Este gran desafío de continuidad en un ambiente turbulento, ha llevado a las empresas a no poder sostener su propuesta en el tiempo. Dentro de este grupo de organizaciones nos encontramos las microempresas cuyo impacto en la sociedad es grande en su conjunto, pero su tamaño individual es ínfimo al tamaño del mercado.

Por lo antes expuesto, el objetivo de la presente investigación es determinar el grado de competitividad de las microempresas según la percepción de sus representantes en el Cantón Manta - Ecuador, siendo esta ciudad una de las principales del país en cuanto a cantidad de empresas, aporte al empleo, comercio nacional y, turismo.

Para comprender a la complejidad de las empresas más pequeñas de la economía, es adecuado comenzar con un análisis de estas organizaciones desde un enfoque de similitudes en la región hispanohablante, por lo que primeramente se reconoce a las microempresas en Latinoamérica para luego acercarse a la realidad en el Ecuador.

Al analizar de forma general a las microempresas en Latinoamérica, siguiendo la investigación de Neira Orjuela (2006) se observa la necesidad de un estudio diferencial y particularizado de este sector, debido a sus características particulares de constitución (muchas veces familiar) no planificado, informal, etc., pero con gran impacto principalmente en lo social y económico a nivel local. En algunas investigaciones como la de Estrada, García \& Sánchez (2009) se estudió la importancia de las microempresas por el aporte a la economía y a la empleabilidad nacional, con al menos el 30.91\% de los trabajadores, como promedio en los países de la región. Saavedra (2012) reconoce la contribución fundamental en lo social económico de todo lo que involucra la existencia de estas organizaciones, aunque expone que la supervivencia es uno de los puntos más críticos del sector; yendo más allá en su investigación se observa la contribución de este grupo de empresas como herramienta de movilidad social no solo por la cantidad de influencia en el empleo de las naciones, sino también por la descentralización de la producción hacia lugares rurales y la no centralización en las grandes urbes. Coincidente con la idea anterior Ortiz (2011) determinó la necesidad de crear un modelo que acentúe la importancia de estas organizaciones en el entretejido 
económico- social de los países latinoamericanos, y el poder multiplicador que estos tienen. Sumado a lo anterior, es de fundamental importancia la creación de pequeños negocios para el sustento de su familia largo plazo con la inclusión de la mujer como sustento fundamental (Main \& Bachrach, 1998).

Además de las características de aporte al empleo y al PBI de los países, las empresas objeto de análisis, también contribuyen a la redistribución de ingresos, la expansión y diversificación de la producción, entre otros (Bradot, 1999). Otras características de las microempresas y que son desfavorables, además de la comprobada dificultad de supervivencia (Guaipatín, 2003) poseen una particular gobernabilidad caracterizada por familias dentro de su estructura de creación y dirección, baja profesionalización de todos los niveles organizacionales, informalidad, contratación de mano de obra sin calificación, por nombrar los más destacados (Barquero, 2003). Así mismo, autores como Arroyo Venegas (2008) indagaron en cuestiones culturales y de desarrollo particularizados en la región, que se vinculan con una idiosincrasia particular de la región como el paternalismo, el enfoque en la conservación de las relaciones sobre los negocios, la dirección familiar y su contratación bajo el mismo principio, incluso las raíces heredadas de la conquista, demostrado por un "lenguaje indirecto" que afecta la ejecución y planteamientos de objetivos.

En general, las microempresas Latinoamericanas tienen sus particularidades, pero debido al aporte en general a los países de la región, es imprescindible su estudio. Al revisar la investigación comparativa entre Latinoamérica, Europa y China de, Niño-Amézquita, Dubrovsky \& Jankurová (2017) se distingue la diferenciación de sectores y regiones que conforman el sector empresarial y lo distinguen con circunstancias y características diferentes, y como tales necesarias de ser estudiadas con sus particularidades.

En lo que respecta a Ecuador, la información oficial sobre el sector productivo es recopilada a través del Instituto Nacional de Estadística y Censos (INEC) y existen 884236 empresas y, además de 1039537 establecimientos. La empresa fue definida por Urmeneta (2016) como una "Unidad organizativa que realiza actividades económicas en uno o varios lugares, con autonomía en la toma de decisiones de mercadeo, financiamiento e inversión. La empresa puede corresponder a una única unidad legal” (p. 8).

La definición de establecimiento, de acuerdo con el Instituto Nacional de Estadística y Geografía de México es:

Unidad económica que, en una sola ubicación física, asentada en un lugar de manera permanente combina acciones y recursos bajo el control de una sola entidad propietaria o controladora, para realizar actividades de producción de bienes, 
compraventa de mercancías o prestación de servicios con o sin fines de lucro, en el que sólo se considera una actividad productiva que es la actividad económica principal (INEGI, 2018, p. 8).

Considerando estas definiciones es que se puede entender la dimensión que tienen las empresas y los establecimientos, y que el INEC (2018) utilizó para la subdivisión de las empresas. A continuación, se muestra el tipo de empresa y establecimientos según el tamaño de estas, que se determina por sus ventas y el número de empleados:

- Grandes: Ventas \$5000001; Personal 200 en adelante

- Medianas “B”: Ventas \$2000001 a \$5000000; Personal 100 a 199

- Medianas “A”: Ventas \$100000 a \$2000000; Personal 50 a 99

- Pequeñas: Ventas \$100001 a \$1000000; Personal 10 a 49

- Microempresas: Ventas menor o igual a \$1000000; Personal 1 a 9

\section{Metodología}

El enfoque de este estudio es cuantitativo de alcance descriptivo, en cuanto se describe el fenómeno del proceso decisorio en el contexto de las microempresas que involucra solamente a personas dentro de las organizaciones de análisis.

El diseño es transversal ya que los datos fueron tomados en un solo momento, mediante una encuesta dirigida al personal encargado o dueños de los negocios.

En cuanto a la fiabilidad de la información, se realizó una búsqueda de información a través de organismos oficiales como el Banco Mundial y el INEC, así como en revistas científicas. En ambos casos se buscó recolectar información que describa de la manera más certera lo que sucede en el sector de análisis.

Respecto al instrumento de medición se planteó la necesidad de una adaptación al modelo de Saavedra et al (2013), el cual mide con más amplitud a las PYMES, es por ello se tomó siete de las ocho variables utilizadas en el modelo base y a partir de ello se indagó sobre las características distintivas y ajustadas a las microempresas:

1. Planeación Estratégica: Proceso de Planeación Estratégica e Implementación de la Estrategia

2. Producción y Operaciones: Planificación y Proceso de Producción, y Manejo de Inventarios

3. Aseguramiento de la Calidad: Aspectos Generales de la Calidad y Sistema de Calidad

4. Comercialización: Mercado Nacional, y Mercadeo y Ventas

5. Contabilidad y Finanzas: Monitoreo de Costos y Contabilidad, y Administración Financiera

6. Recursos Humanos: Aspectos Generales y Cultura Organizacional 
7. Sistemas de Información: Planeación del Sistema y Proceso de Entradas Respecto a la dimensión o área de Gestión Ambiental, no fue incorporada en cuanto no es pertinente según el tipo de organización objeto de análisis. En el estudio del Banco Mundial (2013) se determino la poca importancia que le las microempresas tienen hacia el medio ambiente en general, motivados por no considerar que tenga un impacto directo sobre su negocio.

Cabe aclarar que la medición de la percepción sobre el nivel de competitividad de las microempresas en Manta se realizó a través de 21 preguntas que abarcaron siete variables y 21 subvariables.

Para establecer la fiabilidad del instrumento de medición utilizado en la presente investigación se comprueba mediante el coeficiente Alfa de Cronbach, con un valor de 0.817, valor que demuestra la confiabilidad.

Para determinar la población se parte de los datos oficiales del INEC (2017) que reconoce un total de 78638 empresas en la provincia de Manabí, siendo consideradas de ese total 73724 microempresas. En el cantón Manta se asientan 13,58\% de estas organizaciones, de acuerdo con la investigación de Baque Cantos et al. (2018) determinado una población objeto de estudio de 10012 microempresas. A su vez, para la determinación de la muestra se utilizó el criterio no probabilístico y se estratificó en las 4 zonas que más agrupan negocios pequeños en Manta, quedando la muestra conformada de la siguiente forma:

Los Esteros, 112

Calle 13 y avenida 24, 118

Centro y sector La Bahía, 133

Nuevo Tarqui, 128

Total, de encuestas, 491

\section{Resultados y discusión}

De acuerdo con la información tomada mediante el instrumento aplicado se realiza un análisis de cada variable y subvariable a fin de describir cada una de ellas por separado y poder luego concluir sobre la temática en general. También se incluirá en esta discusión datos extraídos de la recolección de información necesaria para la comprensión de la temática.

Un dato de suma importancia se refiere a la cantidad de empresas y establecimientos en cuanto al tamaño, la estadística recogida determina que solo el $0,43 \%$ de las empresas son Grandes, seguidas con un $0,63 \%$ de las conocidas como Medianas B, un 0,94\% de las denominadas Mediana A, un 7,22\% de Pequeñas empresas y, por último, una amplia mayoría de $90,78 \%$ de las Microempresas. Otra estadística que arroja el análisis del panorama general 
de los Censos Económicos 2018 (INEC, 2018) se refiere a la concentración de las empresas en 5 provincias (con un 62,17\%), encabezada esta lista por Guayas (el puerto más importante del país se encuentra en ella) y Pichincha (con su capital Quito), seguidas por una tercera provincia en cantidad de empresas y/o establecimientos que es Manabí, que incluye el puerto de Manta.

Respecto a la caracterización, sería lógico esperar una relación directa entre tamaño de empresa y aporte, pero la encuesta del INEC (2018) no se corresponde con esta relación en cuanto a la participación de las ventas y el tipo de empresa, el 0,5\% de empresas grandes vende el $71,33 \%$ de lo que circula en el Ecuador, seguida por las pequeñas empresas con un $11,72 \%$, un $9,71 \%$ de empresas medianas tipo $\mathrm{B}$, un $6,28 \%$ de medianas tipo A, con un solo $0,96 \%$ de las microempresas que son más del $90 \%$ de las empresas o establecimientos del país. Se observa un fenómeno contradictorio en la relación venta versus cantidad de microempresas, ya que siendo la mayoría absoluta en cantidad de empresas el aporte es casi del $1 \%$ de la economía. Una de las explicaciones del porqué de la diferencia, es la realidad de las empresas más pequeñas en el Ecuador, que tienden a la informalidad para poder competir con otras organizaciones de mayor tamaño (Talledo Villavicencio \& Solórzano León, 2013). Incluso el Banco Mundial (2013) advierte que casi el 80\% de las micro y pequeñas empresas son informales total o parcialmente (están inscritas, pero no registan la mayoría de sus operaciones) incluso detalla que "las empresas informales emplearon el 37\% de la población ocupada en 2011, frente al $42 \%$ en el 2000. Asimismo, el $62 \%$ de los trabajadores no cubiertos por la seguridad social representa el cuarto porcentaje más alto en América Latina" (p. 13).

Muchas de las particularidades de la informalidad que el Banco Mundial (2013) determinó, conjuntamente con los criterios de Talledo \& Solórzano (2013) necesitan de un estudio particularizado para el sector de la microempresas. Por tal razón se adapta una herramienta con base en el instrumento creado por Saavedra et al (2013) el mismo que estudia a las pequeñas y medianas empresas en Latinoamérica, este cuestionario se enfoca en ocho dimensiones o áreas principales: "1. Planeación estratégica, 2. Producción y compras, 3. Aseguramiento de la Calidad, 4. Comercialización, 5. Contabilidad y Finanzas, 6. Recursos humanos, 7. Gestión ambiental, y 8. Sistemas de información” (Saavedra et al, 2013).

Al observar el ámbito social y el aporte al trabajo que dan a la sociedad las microempresas y de acuerdo con los datos formales del INEC (2018) se observa que el personal afiliado tiene una tendencia casi proporcional entre Grandes empresas $(38,83 \%)$, Pequeñas y Medianas empresas $(36,66 \%)$ y Microempresas $(24,51 \%)$. Uniendo estos datos con el aporte en 
millones de dólares que cada tipo de empresa da como Masa Salarial: 54,67\% Grandes, 30,31\% para las PYMES y 15,02\% Microempresas (representan 4628 millones de dólares de salario de las PYMES a sus trabajadores). Los números reflejan el aporte en lo formal de las más pequeñas organizaciones hacia la sociedad y el empleo, recordando que los datos informales ampliarían exponencialmente los números de las microempresas.

Otros aspectos del sector de acuerdo con el Banco Mundial (2013) y World Bank (2012) reflejan otros aspectos caraterizantes, algunos derivados de la informalidad, como es la baja contribución a las arcas del gobierno a través del impuesto, el escaso acceso al crédito (al no poder demostrar sus ingresos en modo formal), la mezcla entre negocio y familia, la rotación continua de personal, baja capacitación y educación de los involucrados en la microempresa, las malas condiciones estructurales del negocio, la no aplicalidad de los principios de cuidados al medio ambiente, la poca aplicalidad de los prinicipios plasmados en la Ley de Economía Popular y Solidaria, y la limitación de crear flujos constantes de ingresos (aunque el promedio de supervivencia es mayor que el promedio de la región). En el mismo sentido Berger \& Guillamon (2017) determinaron un acercamiento a formas menos formalizadas de acceso al credito y el complemento existente entre las microempresas y las organizaciones no gubernamentales que apoyan el crecimiento y continuidad en el tiempo.

La variable central de esta investigación es la competitividad, dejando de lado el término en el sentido económico o el que se refiere a las naciones o países, para enfocarlo en el empresarial y en el de las micro, pequeñas y medianas empresas, por lo que es correcto empezar entendiendo cuál es el enfoque que se le da a este término, para Saavedra, Milla \& Tapia (2013) que han medido la competitividad empresarial, y enfocan el término como la capacidad de la empresa para mantener o incrementar en base a estrategias empresariales la participación que poseen en el mercado. Además, los propios autores determinan que "la competitividad de una empresa depende de la productividad, la rentabilidad, la posición competitiva, la participación en el mercado interno y externo, las relaciones interempresariales, el sector, el entorno macroeconómico y la infraestructura regional" (p.5). Estas características han sido medidas en Latinoamérica a través de herramientas complejas que miden la competitividad empresarial de grandes empresas y en el sector PYMES a fin de reconocer la situación de estas, pero con la particularidad de no medir a las microempresas. Con herramientas probadas, como el de la Comisión Económica para América Latina y el Caribe (utilizada para medir los elementos de la competitividad externos) y el mapa interno de la competitividad del Banco Interamericano de Desarrollo, se ha medido el sector de las 
pequeñas y medianas empresas, siendo el sector más cercano, se busca adaptar estos instrumentos comprobados y adaptarlos a la realidad socio económica del cantón Manta.

La descripción de datos anteriores en el Ecuador tiene una correlación con las variables de estudio en campo dentro del Cantón Manta. Observando que muchos de las características del sector en Latinoamérica y en Ecuador se observan también en el sector de estudio

Respecto a la primera variable de: Planeación Estratégica, se realizaron tres preguntas, las dos primeras para conocer sobre el proceso de planeación estratégica y una para la implementación de ésta.

La primera pregunta tuvo como objetivo determinar si el dueño o responsable de la microempresa planificaba estratégicamente y si lo ha hecho en los últimos dos años. En cuanto a la segunda pregunta se analizó la frecuencia con la que se analiza el sector donde se está compitiendo a fin de ser más asertivo en la estrategia planificada del negocio. Y, por último, se realizó una tercera pregunta enfocada a la implementación de la planificación y el involucramiento en el logro de los objetivos para lograrlos.

Como se observa en la Tabla 1, se denota un proceder estratégico desordenado: mayoritariamente las microempresas no planifican de una manera formal, aunque se observa a los actores principales del sector a la hora de decidir qué hacer, y se involucra a toda la organización para llevar adelante lo decidido. Lo anterior mostraría una improvisación por parte del negocio, los cuales reaccionan a movimientos de los actores más cercanos a él, como los proveedores o clientes. La relación en cuanto a medición de esta variable coincide con la apreciación de Talledo Villavicencio \& Solórzano León (2013) que lo reconocen como parte de la informalidad del sector y la visión a corto plazo de las microempresas.

Tabla 1: Planificación Estratégica

\begin{tabular}{|l|c|c|c|}
\hline & $\begin{array}{c}\text { Periodicidad en } \\
\text { planeación }\end{array}$ & Análisis del sector & $\begin{array}{c}\text { Involucramiento hacia } \\
\text { los objetivos }\end{array}$ \\
\hline $\begin{array}{l}\text { Totalmente en } \\
\text { desacuerdo }\end{array}$ & $4 \%$ & $2 \%$ & $5 \%$ \\
\hline En desacuerdo & $23 \%$ & $16 \%$ & $14 \%$ \\
\hline $\begin{array}{l}\text { Parcialmente en } \\
\text { Desacuerdo }\end{array}$ & $32 \%$ & $22 \%$ & $21 \%$ \\
\hline $\begin{array}{l}\text { Parcialmente de } \\
\text { acuerdo }\end{array}$ & $21 \%$ & $29 \%$ & $30 \%$ \\
\hline $\begin{array}{l}\text { De acuerdo } \\
\text { Totalmente de acuerdo }\end{array}$ & $7 \%$ & $9 \%$ & $20 \%$ \\
\hline
\end{tabular}




\begin{tabular}{|c|c|c|c|}
\hline & $100 \%$ & $100 \%$ & $100 \%$ \\
\hline
\end{tabular}

Otra variable de análisis es la de Producción y Operaciones, en la cual se realizaron tres preguntas para dos subvariables. Respecto a la primera subvariable de Planificación y procesos de producción, se preguntó si era adecuado el proceso de compra o producción para competir en costos y calidad, y también se analizó si las compras o producción se realizaban en base a una proyección de ventas futuras. En cuanto a la segunda subvariable de Manejo de Inventario, el punto central era encuestar sobre si se maneja una política de negociar con los proveedores los plazos de entrega de materias primas para mantener el inventario en un nivel óptimo.

Según la percepción de los encuestados (Tabla 2) las microempresas son competitivas en lo que respecta a la producción y operación (que muchas veces parte de una compra para reventa). La particularidad se observa respecto al interés mayor en cuanto a la compra o producción y su relación con los costos y calidad, pero un interés un poco menor en la compra planificada por stock mínimo y el motivo de la compra para la continuidad de la venta en el tiempo.

Tabla 2: Producción y operaciones

\begin{tabular}{|c|c|c|c|}
\hline & $\begin{array}{l}\text { Costo y Calidad } \\
\text { Vs, compras o } \\
\text { producción }\end{array}$ & $\begin{array}{lr}\text { Compras } & \mathrm{O} \\
\text { producción } & \text { Vs. } \\
\text { Ventas futuras } & \end{array}$ & $\begin{array}{lr}\text { Política } & \mathrm{de} \\
\text { inventario } & \text { Vs. } \\
\text { Plazos } & \text { con } \\
\text { Proveedores } & \end{array}$ \\
\hline $\begin{array}{l}\text { Totalmente en } \\
\text { desacuerdo }\end{array}$ & $2 \%$ & $1 \%$ & $1 \%$ \\
\hline En desacuerdo & $4 \%$ & $4 \%$ & $6 \%$ \\
\hline $\begin{array}{l}\text { Parcialmente en } \\
\text { Desacuerdo }\end{array}$ & $14 \%$ & $26 \%$ & $26 \%$ \\
\hline $\begin{array}{l}\text { Parcialmente de } \\
\text { acuerdo }\end{array}$ & $23 \%$ & $33 \%$ & $32 \%$ \\
\hline De acuerdo & $25 \%$ & $22 \%$ & $20 \%$ \\
\hline \multirow[t]{2}{*}{$\begin{array}{l}\text { Totalmente de } \\
\text { acuerdo }\end{array}$} & $32 \%$ & $14 \%$ & $15 \%$ \\
\hline & $100 \%$ & $100 \%$ & $100 \%$ \\
\hline
\end{tabular}


La siguiente variable que afecta la competitividad de las microempresas es el Aseguramiento de la Calidad, medido en dos sub variables y cada una de ellas con una pregunta. De este modo, con respecto a los Aspectos Generales de la Calidad, se realizó una pregunta que giraba en torno a la capacitación y mejoramiento continuo en busca de la calidad; y, en lo que respecta al Sistema de calidad, se indagó sobre los mecanismos de la organización para ofrecer mejoras a partir de la necesidad del cliente.

La Tabla 3 muestra que tanto el proceso de mejora para servir mejor al cliente, así como el proceso de mostrar la calidad percibida a través de la capacitación del personal se compensan para seguir una leve tendencia al aseguramiento de la calidad. Por un lado, la mejora continua a través de una retroalimentación positiva hacia los empleados del negocio no es llevada a cabo por la mayoría, dato que en World Bank (2012) determinió del mismo modo en los paises del cono sur de America. No obstante, se intenta adaptar a la necesidad de la clientela a partir de los aportes que ellos dan.

Tabla 3: Aseguramiento de la calidad

\begin{tabular}{|c|c|c|}
\hline & $\begin{array}{l}\text { Capacitación del } \\
\text { personal para la } \\
\text { mejora continua }\end{array}$ & $\begin{array}{l}\text { Proceso de mejoras a } \\
\text { partir de la necesidad } \\
\text { del Cliente }\end{array}$ \\
\hline $\begin{array}{l}\text { Totalmente en } \\
\text { desacuerdo }\end{array}$ & $7 \%$ & $2 \%$ \\
\hline En desacuerdo & $15 \%$ & $6 \%$ \\
\hline $\begin{array}{l}\text { Parcialmente en } \\
\text { Desacuerdo }\end{array}$ & $32 \%$ & $27 \%$ \\
\hline $\begin{array}{l}\text { Parcialmente de } \\
\text { acuerdo }\end{array}$ & $27 \%$ & $30 \%$ \\
\hline De acuerdo & $14 \%$ & $20 \%$ \\
\hline Totalmente de acuerdo & $6 \%$ & $14 \%$ \\
\hline & $100 \%$ & $100 \%$ \\
\hline
\end{tabular}

La cuarta variable analizada es la de Comercialización, enfocada en el Mercadeo y la Venta únicamente, pero con cuatro preguntas: 
1 La empresa tiene claramente definido su mercado objetivo, sus estrategias de penetración, posicionamiento y comercialización.

2 La empresa dispone de información de sus competidores (en cuanto a reputación, calidad de sus productos y servicios, fuerza de ventas y precios).

3 Las estrategias, objetivos y precios de la empresa están determinados con base en sus costos, la oferta, la demanda y la situación competitiva.

4 Los recursos asignados al marketing (material publicitario, promociones, etc.) son adecuados y se usan de manera eficiente.

Las respuestas obtenidas fueron tendientes a la comercialización efectiva a través de los procesos internos de las organizaciones (en las cuatro preguntas las respuestas agrupadas de acuerdo con relación a las en desacuerdo son mayores), pero cabe notar que el conocimiento del mercado objetivo no es conocido por la mayoría, lo que puede producir ineficiencia cuando se quiere acercarse al cliente potencial (tabla 4). El Banco Mundial y Saavedra, Milla \& Tapia (2013) ubican al sector como ineficiente en su modo de mostrar y comercializar los productos y servicios, lo que genera pérdidas por no llegar correctamente al cliente y cubrir parcialmente sus necesidades, muchas veces por desconocimiento de mecanismo de comercialización.

\section{Tabla 4: Comercialización}

\begin{tabular}{|l|c|c|c|c|}
\hline & $\begin{array}{l}\text { Conocimiento } \\
\text { de mercado } \\
\text { objetivo }\end{array}$ & $\begin{array}{l}\text { Conocimiento } \\
\text { de la } \\
\text { competencia }\end{array}$ & $\begin{array}{l}\text { Se } \\
\text { comercializa } \\
\text { a través de } \\
\text { parámetros } \\
\text { objetivos }\end{array}$ & $\begin{array}{l}\text { Eficiencia de } \\
\text { marketing }\end{array}$ \\
\hline $\begin{array}{l}\text { Totalmente } \\
\text { en } \\
\text { desacuerdo }\end{array}$ & $2 \%$ & $2 \%$ & $1 \%$ & $2 \%$ \\
\hline $\begin{array}{l}\text { En } \\
\text { desacuerdo }\end{array}$ & $16 \%$ & $8 \%$ & $6 \%$ & $8 \%$ \\
\hline $\begin{array}{l}\text { Parcialmente } \\
\text { en }\end{array}$ & $23 \%$ & $18 \%$ & $14 \%$ & $20 \%$ \\
Desacuerdo & & & & \\
\hline Parcialmente & $28 \%$ & $30 \%$ & $32 \%$ & $29 \%$ \\
\hline
\end{tabular}




\begin{tabular}{|l|c|c|c|c|}
\hline de acuerdo & & & & \\
\hline De acuerdo & $21 \%$ & $29 \%$ & $30 \%$ & $28 \%$ \\
\hline $\begin{array}{l}\text { Totalmente } \\
\text { de acuerdo }\end{array}$ & $9 \%$ & $13 \%$ & $17 \%$ & $13 \%$ \\
\hline & $100 \%$ & $100 \%$ & $100 \%$ & $100 \%$ \\
\hline
\end{tabular}

En lo que respecta a la variable de Contabilidad y Finanzas, que muestra el orden en las cuentas y el dinero de las microempresas se preguntaron sobre tres temas: sobre la periodicidad del reporte de cuentas por cobrar, a fin de medir la sub variable de Monitoreo de Costo y Contabilidad y dos preguntas sobre si lleva una formalidad en su planeación financiera y si la empresa conoce la rentabilidad de cada producto o línea de producto.

La variable de Contabilidad y Finanzas que se observa en la Tabla 5, muestra una dispersión de las respuestas dadas. Respecto a el caso de las cuentas a cobrar las respuestas tiene un grado mayor de no dar seguimiento a sus cuentas cobrar, aunque la planificación financiera y el conocimiento de rentabilidad por producto (en mayor grado este último) tiene una mayor tendencia hacia la realización y conocimiento de estas. La importancia de esta variable unida a la informalidad general del sector contribuye al poco acceso al sector crediticio (Berger \& Guillamon, 2017) y haciendo que el crecimiento de los negocios sea relentizado, al no poseer sino recursos propios o de familiares o de amigos.

Tabla 5: Contabilidad y Finanzas

\begin{tabular}{|c|c|c|c|}
\hline & $\begin{array}{l}\text { Periodicidad de } \\
\text { reportes de cuentas } \\
\text { a cobrar }\end{array}$ & $\begin{array}{l}\text { Planeación } \\
\text { financiera formal }\end{array}$ & $\begin{array}{l}\text { Rentabilidad de } \\
\text { producto o línea }\end{array}$ \\
\hline $\begin{array}{l}\text { Totalmente en } \\
\text { desacuerdo }\end{array}$ & $4 \%$ & $2 \%$ & $2 \%$ \\
\hline En desacuerdo & $21 \%$ & $12 \%$ & $6 \%$ \\
\hline $\begin{array}{l}\text { Parcialmente en } \\
\text { Desacuerdo }\end{array}$ & $27 \%$ & $28 \%$ & $15 \%$ \\
\hline $\begin{array}{l}\text { Parcialmente de } \\
\text { acuerdo }\end{array}$ & $24 \%$ & $27 \%$ & $34 \%$ \\
\hline De acuerdo & $14 \%$ & $19 \%$ & $30 \%$ \\
\hline \multirow[t]{2}{*}{$\begin{array}{l}\text { Totalmente de } \\
\text { acuerdo }\end{array}$} & $9 \%$ & $11 \%$ & $13 \%$ \\
\hline & $100 \%$ & $100 \%$ & $100 \%$ \\
\hline
\end{tabular}


La penúltima variable sobre el grado de competitividad es sobre Recursos Humanos, medido en dos sub variables con dos preguntas cada una de ellas. Respecto a Aspectos Generales de Recursos Humanos, se preguntó sobre si el personal se maneja por políticas y manuales de procedimientos, y si se cumplen con los todos los requisitos formales en cuanto a personal. Por otra parte, en lo que respecta a Cultura Organizacional, se buscaron respuestas con respecto a la buena comunicación oral y escrita en toda la organización, y si se tiene establecidas actividades, programas e incentivos para mejorar el clima laboral. La Tabla 6 indica los resultados de esta variable, claramente se ve que las dos sub variables tienen un comportamiento opuesto. Respecto a los Aspectos Generales de Recursos Humanos se observa la informalidad del sector, al contratar personal sin cumplir todos los requisitos legales, y no dar indicaciones claras de la forma de proceder dentro de la organización. En el lado positivo se encuentra la sub variable de Cultura Organizacional, cuyas respuestas en ambas preguntas denotan la intención hacia la creación de una cultura que apoye las actividades de la micro empresa.

Tabla 6: Recursos Humanos

\begin{tabular}{|c|c|c|c|c|}
\hline & $\begin{array}{l}\text { Políticas y } \\
\text { manuales de } \\
\text { procedimientos }\end{array}$ & $\begin{array}{l}\text { Cumplimiento } \\
\text { de requisitos } \\
\text { formales }\end{array}$ & $\begin{array}{l}\text { Buena } \\
\text { comunicación } \\
\text { oral y escrita }\end{array}$ & $\begin{array}{l}\text { Mejora de clima } \\
\text { con incentivos }\end{array}$ \\
\hline $\begin{array}{l}\text { Totalmente en } \\
\text { desacuerdo }\end{array}$ & $8 \%$ & $9 \%$ & $2 \%$ & $4 \%$ \\
\hline En desacuerdo & $15 \%$ & $17 \%$ & $10 \%$ & $11 \%$ \\
\hline $\begin{array}{l}\text { Parcialmente en } \\
\text { Desacuerdo }\end{array}$ & $34 \%$ & $36 \%$ & $24 \%$ & $17 \%$ \\
\hline $\begin{array}{l}\text { Parcialmente de } \\
\text { acuerdo }\end{array}$ & $25 \%$ & $23 \%$ & $30 \%$ & $30 \%$ \\
\hline De acuerdo & $12 \%$ & $10 \%$ & $21 \%$ & $24 \%$ \\
\hline \multirow[t]{2}{*}{$\begin{array}{l}\text { Totalmente de } \\
\text { acuerdo }\end{array}$} & $5 \%$ & $4 \%$ & $13 \%$ & $13 \%$ \\
\hline & $100 \%$ & $100 \%$ & $100 \%$ & $100 \%$ \\
\hline
\end{tabular}

La última variable de estudio es Sistema de información midiendo sobre Planeación del Sistema en la cual se planeó si se utilizan sistemas o programas computarizados para llevar de forma ordenada, precisa y útil los datos económicos de su negocio o demás información de sus actividades; y otra respecto a si se generan y archivan adecuadamente los documentos de 
soporte (comprobantes de egreso, facturas de venta, recibos de caja, facturas, etc.) en las diferentes áreas de la empresa.

La Tabla 7 muestra que la información que se usa en estas organizaciones está sistematizada apropiadamente dentro de la organización, pero de acuerdo a los datos de una manera más manual que en base a programas de apoyo a la gestión de la información, existe un alto grado de generación y archivo de información, pero los sistemas informáticos no son utilizados en todos los casos.

\section{Tabla 7. Sistemas de Información}

\begin{tabular}{|l|c|c|}
\hline & $\begin{array}{l}\text { Uso de sistemas } \\
\text { informático para el manejo } \\
\text { de información }\end{array}$ & $\begin{array}{l}\text { Generación y soporte de } \\
\text { documentos }\end{array}$ \\
\hline Totalmente en desacuerdo & $5 \%$ & $4 \%$ \\
\hline En desacuerdo & $14 \%$ & $13 \%$ \\
\hline $\begin{array}{l}\text { Parcialmente } \\
\text { Desacuerdo }\end{array}$ & $21 \%$ & $30 \%$ \\
\hline Parcialmente de acuerdo & $30 \%$ & $22 \%$ \\
\hline De acuerdo & $20 \%$ & $12 \%$ \\
\hline Totalmente de acuerdo & $10 \%$ & $100 \%$ \\
\hline
\end{tabular}

\section{Conclusiones}

Una vez investigada la temática se pudo determinar que el grado de competitividad de las microempresas del Cantón Manta - Ecuador, según la percepción de sus representantes, es media y han tenido una relación con el estudio teórico en cuanto a la informalidad, baja capacitación, poca planificación, dificultades financieras, entre otros. El comportamiento de los datos demuestra una proporción dos a uno en las respuestas de acuerdo con relación a las de en desacuerdo, midiendo de esta manera una competitividad aceptable por parte de las microempresas en Manta.

Aunque las respuestas obtenidas de los máximos representantes de estas organizaciones demuestran su opinión, se debe indagar en mayor profundidad en cada uno de los parámetros de discusión para dejar de lado cualquier tipo de sesgos personal, que se puede tener al consultar percepciones sin observación de la veracidad en la realidad del día a día de estos negocios. Sumado a esto está la preexistente dificultad de supervivencia y la complejidad de 
las microempresas, denotando un esfuerzo superior para la competitividad de estas, debido a las características y particularidades.

\section{Referencias bibliográficas}

Arroyo Venegas, I. (2008). La cultura y el proceso de globalización en el desarrollo de las pequeñas y medianas empresas latinoamericanas. Revista De Ciencias Económicas, 26(1).

Banco Mundial. (14 de agosto de 2013). Ecuador lucha contra la informalidad para potenciar a las pequeñas empresas. Banco Mundial. Recuperado el 17 de septiembre de 2019, de https://www.bancomundial.org/es/news/feature/2013/08/14/ecuador-fight-againstinformality-to-empower-small-businesses

Bachrach, T \& Main, K. (1998) Women and The False Promise of Microenterprise. Sange Journals. 12(4), 424- 444 https://doi.org/10.1177/089124398012004004

Barquero, I. (2003). El estado y la competitividad dela micro, pequeña y mediana empresa. Colección Cuadernos de Desarrollo Humano Sostenible, 21. Tegucigalpa, Honduras: PNUD.

Baque Cantos, M., Baque Cantos, E., Chiquito Tigua, G. \& Baque Parrales, S. (2018). Microempresas en el Ecuador: Caso ciudad de Manta. Dominio de las Ciencias, 4(1), 619-632.

Berger, M \& Guillamon, M (2017). Microenterprise development in Latin America - a view from the Inter-American Development Bank. Practical Action, 7(3), 89-112. https://doi.org/10.3362/0957-1329.1996.021

Bradot, Nestor P. (1999) Las Pymes Latinoamericanas. México, Publisher

Estrada, R., García, D., \& Sánchez, V. (2009). Factores determinantes del éxito competitivo en la Pyme: Estudio Empírico en México. Revista Venezolana de Gerencia, 14 (46), 169-182.

Guaipatín, C. (2003). Observatorio PYME: Compilación estadística para 12 países de la Región. Washington:

INEC (octubre 2018) Directorio de Empresas y Establecimientos 2017. INEC. Recuperado a partir de https:/www.ecuadorencifras.gob.ec/documentos/webinec/Estadisticas_Economicas/DirectorioEmpresas/Directorio_Empresas_2017/Docume ntos_DIEE_2017/Documentos_DIEE_2017/Principales_Resultados_DIEE_2017.pdf

INEGI (2018) Panorama general de los Censos Económicos 2019. Recuperado a partir de https://www.inegi.org.mx/contenidos/programas/ce/2019/doc/pan_gral_2019.pdf 
Neira Orjuela, Fernando. (2006). Elementos para el estudio de la microempresa latinoamericana. Latinoamérica. Revista de estudios Latinoamericanos, (43), 153-174..

Niño-Amézquita, J; Dubrovsky, V \& Jankurová, A (2017). Innovations and Competitiveness in Regional Development: A Comparison of Latin America, Europe, And China. Czech Journal of Social Sciences Business and Economics, 1, 28-37.

Ortiz, Jaime (2011) "Rethinking the Approach to the Microenterprise Sector in Latin America: An Intergrating Framework," Journal of Microfinance / ESR Review: 3(2),

Saavedra, M. L. (2008). Caracterización e importancia de las PYMES en Latinoamérica: Un estudio comparativo. Actualidad Contable. Faces, 17, 122-134. Mérida (Venezuela).

Saavedra García, M. L. (2012). Una propuesta para la determinación de la competitividad en la pyme latinoamericana. Pensamiento y Gestión (33), 93-124

Saavedra García, M., Milla Toro, S. \& Tapia Sánchez, B. (2013). Determinación de la competitividad de la Pyme en el nivel micro: el caso del Distrito Federal, México. Revista FIR, FAEDPYME International Review, 2 (4), 38-52

Talledo Villavicencio, M. V., \& Solórzano León, D. L. (mayo de 2013). Universidad Politécnica Salesiana. Obtenido de Universidad Politécnica Salesiana: https://dspace.ups.edu.ec/bitstream/123456789/4689/1/UPS-GT000420.pdf

Urmeneta, R. (2016) Dinámica de las empresas exportadoras en América Latina. CEPAL

World Bank (2012). Ecuador - The faces of informality: Ecuador - Las caras de la informalidad (Spanish). Washington DC: World Bank. http://documents.worldbank.org/curated/en/942561468246331465/Ecuador-Las-carasde-la-informalida 
Anexos:

\begin{tabular}{|l|l|}
\hline $\begin{array}{l}\text { Realizado } \\
\text { por: }\end{array}$ & \\
\hline Objetivo: & Medir el grado de competitividad de las micro empresas de Manta \\
\hline Instrucción: & $\begin{array}{l}\text { En el siguiente Cuestionario escriba su respuesta o marque con un } \\
\text { círculo o una marca en su respuesta }\end{array}$ \\
\hline
\end{tabular}

Sexo: M: $F$ : Edad:

\begin{tabular}{|c|c|c|c|c|c|c|c|}
\hline $\mathbf{N}$ & $\begin{array}{l}\text { De acuerdo con las siguientes afirmaciones, responda } \\
\text { con la graduación siguiente. }\end{array}$ & 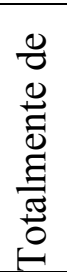 & 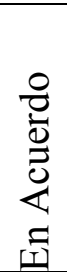 & 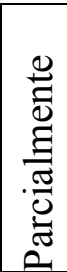 & 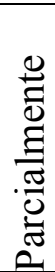 & 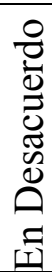 & 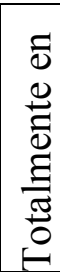 \\
\hline 1 & $\begin{array}{l}\text { Su negocio ha realizado un proceso estratégico de } \\
\text { planeación en los últimos dos años. }\end{array}$ & 6 & 5 & 4 & 3 & 2 & 1 \\
\hline 2 & $\begin{array}{l}\text { Se analiza con frecuencia el sector donde opera la } \\
\text { empresa considerando entre otros factores: nuevos } \\
\text { proveedores, nuevos clientes, nuevos competidores, } \\
\text { nuevos productos competidores, nuevas tecnologías y } \\
\text { nuevas regulaciones. }\end{array}$ & 6 & 5 & 4 & 3 & 2 & 1 \\
\hline 3 & $\begin{array}{l}\text { El personal está activamente involucrado en el logro de } \\
\text { los objetivos de la empresa, así como en los cambios } \\
\text { necesario para lograrlos. }\end{array}$ & 6 & 5 & 4 & 3 & 2 & 1 \\
\hline 4 & $\begin{array}{l}\text { El proceso de producción o compra de la empresa es } \\
\text { adecuado para ofrecer productos con calidad y costos } \\
\text { competitivos. }\end{array}$ & 6 & 5 & 4 & 3 & 2 & 1 \\
\hline 5 & $\begin{array}{l}\text { La planeación de la producción o compra está basada en } \\
\text { pronósticos de ventas futuras o proyectadas. }\end{array}$ & 6 & 5 & 4 & 3 & 2 & 1 \\
\hline 6 & $\begin{array}{l}\text { La empresa tiene como política negociar con los } \\
\text { proveedores los plazos de entrega de materias primas } \\
\text { para mantener el inventario en un nivel óptimo. }\end{array}$ & 6 & 5 & 4 & 3 & 2 & 1 \\
\hline 7 & $\begin{array}{l}\text { Su organización se preocupa por la calidad en la empresa } \\
\text { y para ello capacita adecuadamente a todos los } \\
\text { empleados en aspectos de calidad y de mejoramiento }\end{array}$ & 6 & 5 & 4 & 3 & 2 & 1 \\
\hline
\end{tabular}




\begin{tabular}{|c|c|c|c|c|c|c|c|}
\hline & continuo. & & & & & & \\
\hline 8 & $\begin{array}{l}\text { El sistema de seguimiento o medición de calidad } \\
\text { identifica las necesidades del cliente y lo compara con el } \\
\text { producto o servicio ofrecido por la empresa para } \\
\text { proponer mejoras. }\end{array}$ & 6 & 5 & 4 & 3 & 2 & 1 \\
\hline 9 & $\begin{array}{l}\text { La empresa tiene claramente definido su mercado } \\
\text { objetivo, sus estrategias de penetración, posicionamiento } \\
\text { y comercialización. }\end{array}$ & 6 & 5 & 4 & 3 & 2 & 1 \\
\hline 10 & $\begin{array}{l}\text { La empresa dispone de información de sus competidores } \\
\text { (en cuanto a reputación, calidad de sus productos y } \\
\text { servicios, fuerza de ventas y precios). }\end{array}$ & 6 & 5 & 4 & 3 & 2 & 1 \\
\hline 11 & $\begin{array}{l}\text { Las estrategias, objetivos y precios de la empresa están } \\
\text { determinados con base en sus costos, la oferta, la } \\
\text { demanda y la situación competitiva. }\end{array}$ & 6 & 5 & 4 & 3 & 2 & 1 \\
\hline 12 & $\begin{array}{l}\text { Los recursos asignados al marketing (material } \\
\text { publicitario, promociones, etc.) son adecuados y se usan } \\
\text { de manera eficiente. }\end{array}$ & 6 & 5 & 4 & 3 & 2 & 1 \\
\hline 13 & $\begin{array}{l}\text { Periódicamente (quincenal o mensualmente) se preparan } \\
\text { reportes de cuentas por cobrar, organizados por períodos } \\
\text { de antigüedad. }\end{array}$ & 6 & 5 & 4 & 3 & 2 & 1 \\
\hline 14 & $\begin{array}{l}\text { La empresa tiene una planeación financiera formal } \\
\text { (presupuestos de ingresos y egresos, flujos de caja, } \\
\text { razones financieras, punto de equilibrio, etc.). }\end{array}$ & 6 & 5 & 4 & 3 & 2 & 1 \\
\hline 15 & $\begin{array}{l}\text { La empresa conoce la rentabilidad de cada producto o } \\
\text { línea de producto. }\end{array}$ & 6 & 5 & 4 & 3 & 2 & 1 \\
\hline 16 & $\begin{array}{l}\text { La empresa tiene un políticas y manuales de } \\
\text { procedimientos por todo el personal. }\end{array}$ & 6 & 5 & 4 & 3 & 2 & 1 \\
\hline 17 & $\begin{array}{l}\text { La empresa cumple con todos los requisitos legales } \\
\text { vigentes, considerando organismos municipales y estado } \\
\text { nacional. }\end{array}$ & 6 & 5 & 4 & 3 & 2 & 1 \\
\hline 18 & $\begin{array}{l}\text { Existe una buena comunicación oral y escrita a través de } \\
\text { los diferentes niveles de la compañía. }\end{array}$ & 6 & 5 & 4 & 3 & 2 & 1 \\
\hline 19 & La empresa ha establecido actividades, programas e & 6 & 5 & 4 & 3 & 2 & 1 \\
\hline
\end{tabular}




\begin{tabular}{|l|l|c|c|c|c|c|c|}
\hline 20 & $\begin{array}{l}\text { incentivos para mejorar el clima laboral. } \\
\text { Utiliza sistemas o programas de computadora para llevar } \\
\text { en forma ordenada, precisas y útil los datos dinerarios de } \\
\text { su negocio o demás información de sus actividades. }\end{array}$ & 6 & 5 & 4 & 3 & 2 & 1 \\
\hline 21 & $\begin{array}{l}\text { Se generan y archivan adecuadamente los documentos } \\
\text { de soporte (comprobantes de egreso, facturas de venta, } \\
\text { recibos de caja, facturas, etc.) en las diferentes áreas de } \\
\text { la empresa. }\end{array}$ & 6 & 5 & 4 & 3 & 2 & 1 \\
\hline
\end{tabular}

